\title{
Modos de enseñanza en los videotutoriales de matemáticas: equilibrio entre eficacia puntual y utilidad formativa
}

\author{
Teaching modes in mathematical instructional videos: balance between \\ specific effectiveness and educational utility
}

\author{
Claudia Margarita Acuña Soto* \\ ORCID iD 0000-0003-3828-0557 \\ Vicente Liern ${ }^{* *}$ \\ ORCID iD 0000-0001-5883-9640
}

\begin{abstract}
Resumen
Presentamos una propuesta de análisis de videotutoriales de internet basado en los estilos cognitivos Holísticoanalítico y Verbal-imaginativo. Para ello, se distinguen entre dos modos de enseñanza: la eficacia puntual (resolver un problema aquí y ahora) y la utilidad formativa (resolver un problema ahora y para problemáticas afines). La utilidad docente de un videotutorial puede depender de un equilibrio adecuado entre estos modos de enseñanza. Se trata de lograr resolver un problema ahora, pero con sustentos generales y a más largo plazo. La caracterización de los videotutoriales, en este sentido, podría ayudar a los profesores en la elección de videos, ya que según sus necesidades, las decisiones deberían ser diferentes. Sin embargo, no sólo debemos tener en cuenta el punto de vista del docente, sino el potencial incremento en la participación activa y autónoma del estudiante usando los videos adecuadamente elegidos. Para mostrar la aplicación de nuestro análisis, comparamos la producción de tres autores de habla hispana muy reconocidos, que ejercen gran influencia en la actualidad. A través del análisis de varios videos, que puede apoyarse en métodos cuantitativos, se exponen algunos efectos de los modos de enseñanza en la formación matemática.
\end{abstract}

Palabras clave: Modos de enseñanza. Estilos cognitivos. Tratamiento del discurso. Videotutoriales. Materiales.

\begin{abstract}
We present a proposal for the analysis of Internet video tutorials based on the Holistic-analytical and Verbalimaginative cognitive styles. We will distinguish between aspects of teaching modes: Specific effectiveness (solving a problem here and now) and a Educational utility (solving a problem now and for related problems). From those aspects, we suggest the idea of the balance degree between them, that is, to solve a problem now, but with general and longer-term support. The characterization of video tutorials in different teaching modes could help the teachers' choice of videos, according to their needs, decisions should be different. Besides we must not only consider the teachers' view, but the potential increase in active and autonomous participation of students using properly chosen videos. To show the implication of our analysis, we compare the production of videos of well-known Spanish-speaking authors who exert great influence. Through some of this production, we expose the effects of the teaching modes in mathematical education.
\end{abstract}

Keywords: Teaching modes. Cognitive styles. Speech treatment. Educational videos . Materials.

\footnotetext{
* Doctora en Ciencias Pedagógicas (Matemáticas), Instituto Superior Enrique José Varona (ISPEJV). Investigadora en Matemática Educativa, Centro de Investigaciones y de Estudios Avanzados del Instituto Politécnico Nacional (CINVESTAV), C. De México, México. Dirección postal: Av. Instituto Politécnico Nacional, No. 2508, Col. San Pedro Zacatenco, Gustavo A. Madero, 07360, México. E-mail: claudiamargarita_as@ @otmail.com.

*** Doctor en Ciencias Matemáticas (Física Teórica), Universitat de València (UV). Catedrático de Matemáticas para la Economía y la Empresa, Universitat de València (UV), Valencia, España. Dirección postal: Facultad de Economía, Avenida de Tarongers, s/n, Valencia, España, 46022. E-mail: vicente.liern@uv.es.
} 


\section{Introducción}

En la actualidad, los estudiantes han incrementado el uso de videotutoriales de matemáticas disponibles en internet como recursos fiables de la educación, situándolos en ocasiones al mismo nivel que los libros de texto e integrándolos, funcionalmente, a la clase de matemáticas (SANTOS MELLADO, 2018). La casi ilimitada disponibilidad de estos materiales ha alcanzado las aulas, donde pueden ser usados para complementar las tareas de los estudiantes (RIVADENEIRA LOOR, 2013), para valorar la actividad docente (KERSTING, 2008), o como recurso para visualizar y comparar la práctica del profesor (BADILLO et al., 2013), entre otros variados objetivos. Ante esta realidad, creemos que es necesario analizar los distintos modos de enseñanza que se utilizan en estas producciones, apoyando estilos cognitivos de pensamiento.

Los videos disponibles en internet no sólo abordan temas matemáticos escolares, sino que el tratamiento que se hace de ellos transmite una concepción refleja distintos modos de enseñanza. Para reconocer las diferencias entre estos modos y entender las bondades y restricciones de cada uno, es crucial permitir al profesor elegir el respaldo adecuado para su clase a corto plazo, como parte de las actividades que le permitirán enfrentar las condiciones de enseñanza-apredizaje actuales (SULLIVAN; MOUSLEY, 2001).

Por otro lado, la utilidad de estos videotutoriales ha sido frecuentemente asociada a la idea de calidad. Por ejemplo, se han elaborado catálogos de autores de videos de la plataforma Youtube que cumplen ciertos estándares para la enseñanza, tales como aspectos técnicos, didácticos, de contenido y de comunicación, entre otros. Por ejemplo, en Romero, Vásquez y Graván (2017), la idea de calidad se asocia a la introducción de mejoras técnico-didácticas y se decide a través de un consenso entre expertos.

Para nosotros, la ides de calidad se asocia a las llamadas Idoneidades Didácticas (GODINO, 2013; BREDA; FONT; PINO-FAN, 2018). Por esta razón, en la presente investigación analizamos los modos de enseñanza en videos de internet representativos, usando una adaptación de las idoneidades mediante indicadores empíricos. Estas idoneidades establecen aspectos necesarios para enseñar matemáticas que se vinculan con la corrección del contenido matemático, su tratamiento didáctico, la comunicación de los contenidos, el uso de medios tecnológicos, la disposición afectiva y la proximidad con los objetivos escolares (según el nivel del estudiante). Precisamente, estas ideas, transformadas en consignas reconocibles que permitan valoración de videos, serán las que hagan posible nuestro estudio. Por ello, necesitamos valorar los productos elegidos respecto de los modos de enseñanza y su potencial aporte como apoyo a la clase de matemáticas, considerando que son materiales que promueven 
un estilo cognitivo particular (KRUTESTKII, 1976; PRESMEG, 1986). A través de determinadas estructuras, estos estilos destacan las habilidades naturales de representación de la información para resolver problemas matemáticos (PITTA-PANTAZI; CHRISTOU, 2009; PITTA-PANTAZI; SOPHOCLEOUS; CHRISTOU, 2013).

Los estudios en este ámbito proponen agrupar estas habilidades en dos grandes bloques: un estilo que aborda el problema como si fuese un todo formado por sus partes (estilo llamado holístico-analítico, HA), y otro estilo para resolver los problemas usando imágenes visuales (estilo verbal-imaginativo, VI). Por supuesto, estas categorías no son disjuntas, sino que hay estudiantes que ponen en funcionamiento habilidades que les permiten representar la información como las partes de un todo, o sustentado por imágenes mentales. Esta actitud bimodal lo que da lugar a un tercer estilo llamado armónico.

El objetivo de este trabajo es plantear que los modos de enseñanza, representados por la eficacia puntual, la utilidad formativa y el equilibrio entre ellas, son instrumentos que determinan la diferencia de los estilos cognitivos promocionados por los videotutoriales y pueden ayudar a los profesores a elegir videos de matemáticas, según sus necesidades. Para mostrar el funcionamiento y alcance de nuestra propuesta, se analizan algunas producciones representativas de autores hispanoparlantes muy reconocidos en la plataforma Youtube.

\section{Antecedentes}

Para usar videos como apoyo en el aula, se requiere saber cómo se usan y con qué frecuencia. Por esta razón, en 2017 nos planteamos la necesidad de realizar una encuesta a estudiantes de la Facultad de Matemáticas de la Universidad de Valencia, España, y la Facultad de Ciencias de la UNAM, México (SANTOS MELLADO, 2018). Las preguntas que se plantearon aparecen en el siguiente cuadro.

\begin{tabular}{|c|c|c|c|c|c|c|}
\hline \multicolumn{7}{|c|}{ Encuesta: Preferencias y uso de videos de matemáticas de internet (UV, UNAM) } \\
\hline \multicolumn{3}{|l|}{ Edad: } & \multicolumn{4}{|c|}{ Grado de estudio: } \\
\hline \multicolumn{3}{|c|}{$\begin{array}{l}\text { 1. ¿Usas videos de internet para la clase de } \\
\text { matemáticas? }\end{array}$} & \multicolumn{2}{|r|}{ SÍ } & \multicolumn{2}{|c|}{ NO } \\
\hline \multicolumn{3}{|c|}{$\begin{array}{l}\text { 2. ¿Buscas un video si tienes un problema con la clase } \\
\text { de matemáticas? }\end{array}$} & \multicolumn{2}{|r|}{ SÍ } & \multicolumn{2}{|c|}{ NO } \\
\hline $\begin{array}{l}\text { 3. Valora cuánto apoyan los videos } \\
\text { tu clase de matemáticas (siendo } 0 \\
\text { nada y } 10 \text { mucho) }\end{array}$ & $\begin{array}{l}{[0,2]} \\
\text { Nada }\end{array}$ & $\begin{array}{c}{[2,4]} \\
\text { Algo }\end{array}$ & & \begin{tabular}{l|l}
{$[4,6]$} & \\
Más o & \\
menos & \\
\end{tabular} & $\begin{array}{c}{[6,8]} \\
\text { Suficiente }\end{array}$ & $\begin{array}{l}{[8,10]} \\
\text { Mucho }\end{array}$ \\
\hline 4. ¿Cómo prefieres el video? & \multicolumn{2}{|c|}{$\begin{array}{l}\text { Con definiciones } \\
\text { y resultados }\end{array}$} & eje & $\begin{array}{l}\text { n muchos } \\
\text { os y detalles }\end{array}$ & \multicolumn{2}{|c|}{ Que aclare dudas } \\
\hline $\begin{array}{l}\text { 5. ¿Qué esperas de un video de } \\
\text { matemáticas? }\end{array}$ & \multicolumn{2}{|c|}{ Un resultado } & & explicación & \multicolumn{2}{|c|}{ Resolver la tarea } \\
\hline
\end{tabular}




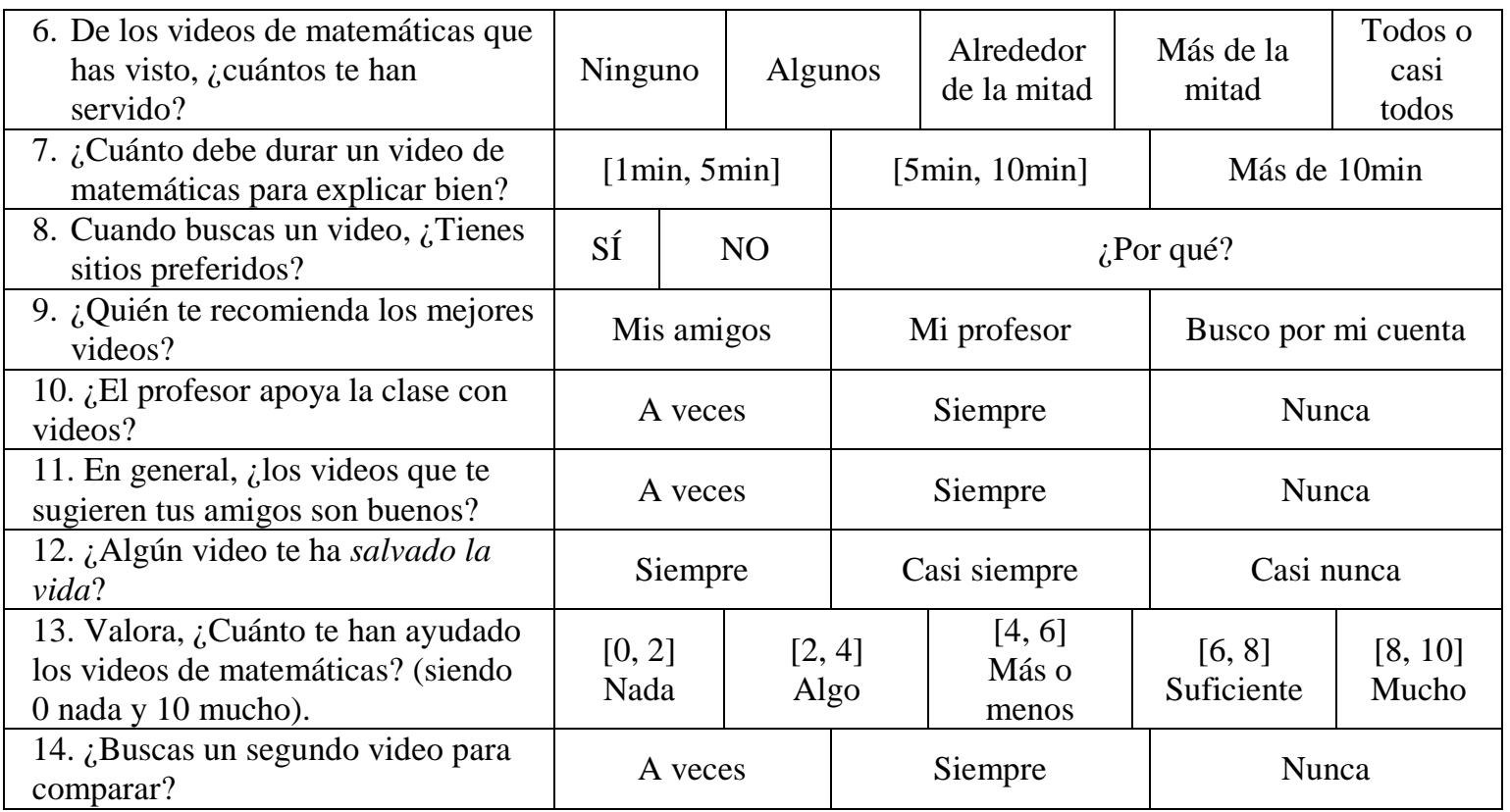

Cuadro 1 - Encuesta sobre preferencias y uso de videotutoriales Fuente: SANTOS MELLADO (2018).

A partir de las opiniones de 45 estudiantes en Valencia (V) y 41 estudiantes en México (M), en Santos Mellado (2018) se deduce que un porcentaje amplio (65.2\% V, 59.2\% M) usa videotutoriales de matemáticas para resolver problemas de clase $(79 \% \mathrm{~V}, 59 \% \mathrm{M})$ y aunque buscan en estos productos obtener una explicación detallada $(93 \% \mathrm{~V}, 100 \% \mathrm{M})$, consideran que reciben un apoyo pobre de ellos. De hecho, solo alrededor de un cuarto de los alumnos cree que el apoyo ha sido suficiente $(25.8 \% \mathrm{~V}, 22.22 \% \mathrm{M})$.

En esta búsqueda, no muchas producciones les fueron útiles para sus clases $(51.1 \% \mathrm{~V}$, $40.7 \% \mathrm{M})$ y las localizaron por su cuenta $(81.3 \% \mathrm{~V}, 62 \% \mathrm{M})$ orientados, fundamentalmente por sus amigos $(69.7 \% \mathrm{~V}, 66.6 \% \mathrm{M})$, ya que sus profesores casi nunca les hacen sugerencias al respecto $(86 \% \mathrm{~V}, 70.3 \% \mathrm{M})$. Estos últimos porcentajes son una muestra más de que la rapidez con la que se incorporan los conocimientos por la vía audiovisual toma por sorpresa a muchos profesores.

Considerando los datos de esta muestra, podríamos suponer que gran parte de los millones de visitas en internet a los videos de matemáticas, las hacen estudiantes por su cuenta, y que las instituciones prácticamente no intervienen en este proceso. Está claro que es necesario valorar los videos tutoriales de matemáticas para asegurar la calidad en su consumo, pero, también hay que observar las aportaciones cognitivas que promueven. En consecuencia, en este trabajo se estudiaron las producciones de algunos sitios y plataformas muy solicitados de habla hispana y que son, ya en estos momentos, un referente.

Pese a que actualmente, algunos centros educativos cuentan con una amplia producción de videos elaborados para autoconsumo como parte de su estrategia educativa, (ACUÑA- 
SOTO; LIERN; PÉREZ-GLADISH, 2018a), el consumo masivo se apoya en la producción abierta de internet y se lleva a cabo sin restricciones de país, régimen o filosofía educativa, pero que encontramos en internet, y con cada vez más influencia, entre nuestros estudiantes.

El videotutorial de matemáticas cubre ciertas necesidades de los estudiantes, asociadas a la disponibilidad de información, que por cualquier causa, no ha sido obtenida en clase (SANTOS-MELLADO et al., 2017). Estos materiales tienen algunas ventajas educativas que una clase no puede proveer: está permanentemente disponible, puede repetirse cuantas veces sea necesario, en caso de no cumplir nuestras expectativas, puede elegirse otro más conveniente, lo que eventualmente nos permite valorar sus cualidades y defectos según objetivos particulares.

Pese a la enorme y creciente oferta en internet, la tendencia general en la elaboración de videos de matemáticas tiene objetivos parecidos. Muchas de las producciones pueden considerarse como una clase del tipo disertación magistral, que siguen siendo muy apreciadas por un buen número de nuestros estudiantes y profesores. Por otro lado, es innegable que el consumo de videos estimula la integración de los recursos tecnológicos en los salones de clase (HOHENWATER; KOKADER; HOHENWATER, 2017), podrían mejorar la enseñanza (CHEUNG; SLAVING, 2013; DONEVSK-TODOROVA; TRGALOVA, 2017) o, incluso, intentan mejorar la docencia con la ayuda de estos recursos (ZABALZA et al., 2016).

La valoración de video tutoriales ha impulsado distintas metodologías que consideran aspectos cualitativos, como la profundidad del entendimiento de los estudiantes a través de valoraciones y la discusión de video clips de la clase de matemáticas (SHERIN; LINSENMEIER; van ES, 2009). En el caso que nos ocupa, la valoración de los videos de internet se apoya en el modelo matemático denominado Valoración Fuzzy Multicriterio ValFM, (LABIPE, 2018), que se sustenta en la lógica difusa y un método de optimización multicriterio llamado TOPSIS. Expresado de forma algorímica, los pasos de ValFM son los siguientes:

Inicio: (1) Un conjunto de $n$ videos, $\mathrm{V}_{1}, \mathrm{~V}_{2}, \ldots, \mathrm{V}_{\mathrm{n}}$, valorados en $m$ ideoneidades didácticas. Cada idoneidad puede ser valorada mediante números, expresiones lingüísticas o intervalos numéricos.

(2) Cada idoneidad tiene una importancia relativa (peso) que puede ser determinado por el docente, el equipo docente e incluso por el usuario del vídeo.

Paso 1: Se normalizan las valoraciones convirtiéndolas en números y haciendo que estén en el intervalo [0,1].

Paso 2: Se multiplican las valoraciones normalizadas por los pesos correspondientes a cada idoneidad. 
Paso 4: Se construyen dos soluciones ficticias que constituyen el mejor video posible (tiene la máxima puntuación en todas las idoneidades) $\mathrm{A}^{+}=(1,1, \ldots, 1)$ y el peor video posible (tiene la menor puntuación en todas las idoneidades) $\mathrm{A}^{-}=(0,0, \ldots, 0)$.

Paso 5: Usando la distancia euclídea $d$, se calcula la distancia de cada video a $\mathrm{A}^{+} \mathrm{y} \mathrm{A}^{-}$ $d\left(\mathrm{~V}_{\mathrm{i}}, \mathrm{A}^{+}\right), d\left(\mathrm{~V}_{\mathrm{i}}, \mathrm{A}^{-}\right)$.

Final: se valora globalmente cada video mediante: $R_{i}=\frac{d\left(V_{i}, A^{-}\right)}{d\left(V_{i}, A^{-}\right)+d\left(V_{i}, A^{+}\right)}$.

El modelo ValFM ya ha sido puesto en funcionamiento, por ejemplo, con estudiantes de licenciatura quienes hicieron y valoraron colectivamente sus propios videos (LECHUGA; MARTÍNEZ, 2019). En definitiva, se trata de un proceso en el que hay una valoración que permite cuantificar datos cualitativos $\mathrm{y}$, posteriormente, los videos pueden organizarse (ordenarse, agruparse etc.) de acuerdo con los intereses prefijados.

\section{Marco Referencial}

El estudio de las habilidades requeridas para aprender matemáticas ha sido motivo de investigación desde la época en la que se priorizaban los métodos conductistas, hasta las más actuales aproximaciones cognitivas (KRUTETSKII, 1976). Los estilos cognitivos atienden las diferencias entre tipos de habilidades para representar la información que se pone en funcionamiento cuando los estudiantes se enfrentan a un problema matemático. En particular, en sus extensas investigaciones sobre las tipos y grados de habilidades entre los estudiantes, Krutetskii reportó que: “Como resultado del estudio, basado en los parámetros indicados, todos los examinados fueron divididos en tres grupos [...] esto permite aislar un tipo analítico [...] (mente matemáticamente abstracta) [...] uno geométrico [...] (mentalmente pictórica) y un tipo armónico” (KRUTETSKII, 1976, p. 315).

Establecer cuáles son las habilidades personales para enfrentarse a las problemáticas matemáticas, es un recurso valioso para diseñar una enseñanza que las aproveche, ya que se esperaría que éstas fueran un recurso disponible naturalmente en los estudiantes al resolver problemas. Estas habilidades no solo se pueden aprovechar para mejorar las condiciones de aprendizaje, sino que se reflejan de forma natural en la enseñanza, como Messick (1994) sugiere:

Los estilos cognitivos son espontáneos invocados sin conciencia o elección en una amplia variedad de situaciones que tienen requisitos similares de procesamiento de información en ausencia de señales personales obligadas para actuar de forma distinta [...] Como consecuencia, los estilos cognitivos tienen potencialmente profundas implicaciones para el aprendizaje y la estructuración del conocimiento (MESSICK, 1994, p. 129). 
Por su parte, Pask (1988) propone dos estrategias de aprendizaje apoyadas en dos particulares tipos de habilidades:

Holístico: interesado en predicados y relaciones globales de tópicos y Seriales que prefieren no usar esas relaciones y aprenden paso a paso [...] los aprendices seriales muestran la intención de investigar datos específicos. Los holísticos muestran interés por verificar un gran número de predicados o hipótesis (PASK, 1988, p. 90).

Las categorías de estilos cognitivos analítico-holístico y verbal-imaginativo han sido propuestas por Pitta-Pantazi y Christou (2009), caracterizándolas de la siguiente manera:

\begin{abstract}
La posición de los individuos a lo largo de la dimensión verbal-imaginativa refleja la manera cómo representan la información mientras piensan, ya sea mediante palabras o imágenes mentales, mientras que la dimensión analítica-holística refleja si entiende una situación como un todo o si lo ven en partes. Sin embargo, dado que tanto la Verbal-imaginativa como la Analítica-holística se encuentran en un continuo, se argumenta que hay algunos individuos que no muestran preferencia por el procesamiento, la representación o la estructura ya sea verbal o de imágenes (PITTAPANTAZI; CHRISTOU, 2009, p.6-7).
\end{abstract}

Respecto a las habilidades de visualización matemática, es importante establecer que no se trata solo del uso de diagramas para resolver los problemas, sino de usar las imágenes mentales como recursos de representación y reflexión para resolver problemas. El objetivo es el mismo con el que se usan los recursos procedimentales según Gutiérrez (1996) "Una imagen mental es una representación mental de un concepto matemático o propiedad que contiene información basada en elementos pictóricos, gráficos o diagramáticos. Visualización, o pensamiento visual es un tipo de razonamiento basado en el uso de imágenes visuales" (GUTIERREZ, 1996, p. 6).

Si la visualización es la base del razonamiento puesto en juego para resolver problemas, entonces se hace uso de imágenes visuales para resolver problemas, aunque las representaciones pictóricas no estén directamente involucradas, sino sugeridas. En particular, Presmeg (1986) afirma que

\footnotetext{
Un método visual de solución es aquel que involucra imágenes visuales, con o sin diagramas, como parte esencial del método de solución, incluso si también se emplean métodos de razonamiento o algebraicos. Un método de solución no visual es aquel que no implica imágenes visuales como parte esencial del método de solución (PRESMEG, 1986, p. 298).
}

Por lo tanto, la presencia o no de diagramas no es lo que lo determina el carácter o método visual de resolución de problemas, sino el uso de las imágenes visuales que son consideradas como "esquemas mentales que representan información visual o espacial" (PRESMEG, 1986, p. 297). 


\subsection{Estilos cognitivos y videotutoriales}

En la actividad docente, se reflejan naturalmente los estilos cognitivos mencionados, debido a que, cuando enseñamos, preferentemente usamos el tipo de representaciones que nos son propios. Por ello, cuando un video se usa como clase magistral es posible identificar los estilos cognitivos de sus autores al resolver problemas.

Para analizar los videos bajo esta óptica, vamos a introducir dos modos de enseñanza: la eficacia puntual y la utilidad formativa, así como la armonía entre ellas, a la que denominamos equilibrio formativo. Se estudian las consignas subyacentes para resolver problemas en los videos analizados, es decir la forma particular de representar la información para resolver problemas matemáticos.

El estilo cognitivo analítico-holístico está asociado a la eficacia puntual debido a que se representa la información como un todo o en sus partes, y como modo de enseñanza tiene el efecto de representar un problema de modo autocontenido. Por lo tanto, la consigna asociada a este tipo de producción es resolver el problema aquí y ahora.

El estilo cognitivo visual-imaginativo está asociado al modo de enseñanza utilidad formativa. La información se apoya en el uso de imágenes visuales, estableciendo conexiones con contenidos que van más allá de lo presentado en el problema resuelto. La consigna asociada a este modo de enseñanza es la resolución del problema aquí y para después.

Entre los dos modos de enseñanza anteriores puede darse un equilibrio formativo, considerando el estilo armónico de Krutetskii (1976, p. 315) o el punto intermedio en el continuo cognitivo al que se refieren Pitta-Pantazi y Christou (2009, p. 6-7). En definitiva, se trata de hacer valer la inclinación bimodal de representar la información. En este caso, la consigna asociada al modo de enseñanza es resolver un problema ahora basándose en estructuras matemáticas más generales.

Nuestro trabajo no parte de la contraposición de las aproximaciones procedimentales contra las conceptuales (CASTRO; PRAT; GORGORIÓ, 2016) porque, a nuestro parecer, la actividad procedimental no está exenta de tratamientos conceptuales, ni la actividad que contempla el uso de imágenes visuales carece de tratamientos procedimentales. Consideramos que ambos casos tratan aspectos distintos del mismo cuerpo de conocimiento, pero que, en cada caso, domina alguno de los aspectos mencionados.

En nuestra experiencia, el aprendizaje de la matemática requiere de la eficacia puntual y de la utilidad formativa, y la utilidad en el aula dependerá de un buen equilibrio entre ellos. Estos modos de enseñanza asociados a las estructuras cognitivas dominantes y a consignas 
específicas de procedimiento, permiten decidir al profesor, qué tipo de video es más conveniente, según las necesidades en cada momento particular. Además, esto contribuye a la adquisición de un pensamiento reflexivo apoyado en la toma de decisiones, lo que podría promover una aproximación inquisitiva (SULLIVAN; MOUSLEY, 2001) poniendo a su disposición materiales que incluyen desde el apoyo directo a la docencia hasta el mejoramiento profesional.

\subsection{Epistemología y modos de enseñanza}

Los modos de enseñanza que hemos presentado, pensados como categorías, muestran una diferencia ontológica importante: mientras que para la eficacia puntual la resolución de problemas está autocontenida, para la utilidad formativa se usan imágenes que lo relacionan con otros problemas y otros contenidos matemáticos. Esta diferencia puede suavizarse mediante el equilibrio entre los dos modos: el equilibrio entre ellos permite resolver los casos particulares y se facilita la generalización de forma apropiada.

La epistemológica subyacente al tipo de conocimiento que puede ser promovido en cada modo de enseñanza también es distinta. En la eficacia puntual se privilegia un pensamiento lógico proposicional conjuntivo y de carácter procedimental. Con la utilidad formativa la lógica proposicional de la estructura dominante es disyuntiva, por lo que dominan las problemáticas asociadas. El equilibrio entre ambos modos privilegia la implicación desde el punto de vista de la lógica proposicional, acompañado de un cumplimiento riguroso de definiciones.

Aunque lo videos no pueden sustituir a las clases presenciales, que en nuestra opinión son necesarias por su valor epistemológico en la construcción social del conocimiento (VIGOTSKY, 1978) y el importante recurso de compartir puntos de vista en el salón de clase (HERSHKOWITZ et al. 2007), sí pueden proveer una figura institucional que actúa como profesor y que hace las veces de un mentor de la educación (TALL, 2007). Esto nos lleva a considerarlo como una buena contribución a la actividad docente como apoyo de la clase.

Identificar los modos de enseñanza mencionados requiere de una evaluación previa de los videos en multitud de aspectos. En Santos Mellado y Acuña Soto (2017) se evalúan a través de una adaptación de las llamadas idoneidades didácticas propuestas en el Enfoque Ontosemiótico, EOS, (GODINO, 2013; GODINO; BATANERO; FONT, 2007). En palabras de Godino, Batanero y Font (2007): 
Idoneidad espistémica: grado de representatividad de los signiticados intitucionales implementados (o pretendidos), respecto de un significado de referencia [...]

Idoneidad cognitiva: expresa el grado en que los significados pretendidos/implementados estén en la zona de desarrollo potencial [...]

Idoneidad interaccional: Un proceso de enseñanza-aprendizaje tendrá mayor idoneidad desde el punto de vista interaccional si las configuraciones y trayectorias didácticas permiten, por una parte, identificar conflictos semióticos potenciales [...]y por otra parte permita resolver los conflictos que se producen durante el proceso de instrucción[...].

Idoneidad mediacional, grado de disponibilidad y adecuación de los recursos materiales y temporales necesarios para el desarrollo del proceso de enseñanza aprendizaje.

Idoneidad emocional: grado de implicación... del alumnado en el proceso de estudio[...].

Idoneidad ecológica, grado en el que el objeto de estudio se ajusta al proyecto educativo[...]. (GODINO, BATANERO y FONT, 2007, p. 133)

Existe bastante literatura en la que se utilizan adaptaciones de estas idoneidades como indicadores para valorar la calidad de los videos (SANTOS MELLADO, 2018; ACUÑASOTO; LIERN; PÉREZ-GLADISH, 2018a; 2018b; 2019). Sin embargo, si queremos que el profesor se sienta cómodo utilizando y recomendando videos como herramientas útiles en la docencia, es necesario conocer el modo de enseñanza del producto. Sólo cuando éste y el estilo cognitivo se adapten a sus habilidades personales y a las necesidades de sus alumnos y alumnas el video se habrá convertido en un instrumento eficaz.

Tal y como se hizo con la necesidad de valorar las idoneidades para tener una idea aproximada de la calidad de los videos (ACUÑA-SOTO; LIERN; PÉREZ-GLADISH, 2018b), para tener una estimación de la eficacia puntual, la utilidad formativa y el equilibrio entre ambas, se planteó una simplificación de estos conceptos. Se trata de conocer hasta qué punto se aconsejaría el video para resolver una tarea en casa o cuán apropiado le parece para estudiar la asignatura. En la sección siguiente expresamos estas dos cuestiones mediante dos preguntas.

\section{Metodología}

La presente investigación es de corte cualitativo interpretativo y pretende utilizar los modos de enseñanza como instrumentos de análisis de la producción de videos de varios autores representativos, destacando los estilos cognitivos promovidos por ellos, así como las consignas dominantes sobre la resolución de problemas. Para ello, se tomó una muestra de la producción de tres autores, con un amplia producción, que se ajustan a los modos de enseñanza que se discutió. La elección también se basa en el reconocimiento que tienen en los paises de habla hispana, respaldados por una audiencia que se cuenta por millones de vistas en internet. 
Se tomó un video del autor Julio Alberto Ríos, cuya dirección de internet es Julioprofe o Julioprofenet, (en la plataforma Youtube www.youtube.com/user/julioprofenet), otra producción de David Calle, cuya dirección en internet es Unicoos (www.unicoos.com) en la misma plataforma y, finalmente, tomamos algunos fragmentos de la producción de Juan Medina con dirección en (www.lasmatematicas.es). En adelante, se hizo referencia a estos autores por el nombre de sus respectivas direcciones de internet.

Para acotar la investigación, nuestro análisis se centró en el tratamiento de ecuaciones lineales, asociados a problemas, que pueden encontrarse en las direcciones siguientes:

- Julioprofe: https://www.youtube.com/watch?v=9xqTSkGECo8 (V1)

- Unicoos: https://www.youtube.com/watch?v=XG1bYAqSXbw (V2)

- Lasmatematicas.es: https:/www.youtube.com/watch?v=9e8bhxX74RE https://www.youtube.com/watch?v=y9rNDDnTYWA (V4).

\subsection{Calidad de los videotutoriales}

En otros estudios se han propuesto seis idoneidades o facetas didácticas como indicadores que permiten "elaborar una teoría de diseño instruccional, apropiada para orientar los procesos de enseñanza" (GODINO, 2013, p. 113). Como herramientas educativas, los videotutoriales pueden valorarse en cada una de las seis facetas, por ejemplo a través seis preguntas cortas (ACUÑA-SOTO; LIERN; PÉREZ-GLADISH, 2018a):

1. Faceta epistémica: ¿el contenido matemático es correcto?,

2. Faceta cognitiva: ¿la didáctica es adecuada?,

3. Faceta interaccional: ¿resulta ameno y/o atractivo?,

4. Faceta mediacional: ¿utiliza adecuadamente el tiempo?,

5. Faceta afectiva: ¿el discurso es amigable?,

6. Faceta ecológica: ¿el contenido se ajusta al proyecto educativo?

En Santos Mellado et al. (2018), se propone la valoración numérica (en una escala del 0 al 10) de cada una de las idoneidades y mediante métodos de ordenación multicriterio se obtiene una ordenación de una colección de videos (ACUÑA-SOTO; LIERN; PÉREZGLADISH, 2018a). En este trabajo, nuestro interés no es obtener un ranking de videotutoriales, ni conocer la valoración global que obtiene un video, sino que buscamos conocer las razones por las que a un video se le ha otorgado determinada valoración. Para conseguir nuestro objetivo, la evaluación cuantitativa de estas facetas (SANTOS MELLADO, 2018) no nos aporta información, de ahí que hayamos preferido una breve reflexión cualitativa. Con ello, teniendo 
en cuenta la opinión de varios profesionales de la docencia en cada faceta, obtenemos información que resultará muy útil para nuestro propósito de analizar los modos de enseñanza.

\subsection{Modos de enseñanza promovidos por los videotutoriales}

Para valorar la calidad docente de los videotutoriales existen trabajos que aproximan la valoración mediante la respuesta (cuantitativa o cualitativa) a preguntas breves (ACUÑASOTO; LIERN; PÉREZ-GLADISH, 2018a, 2018b, 2019). De manera similar, para dar más respaldo a nuestras afirmaciones respecto de los modos de enseñanza, creemos que resulta útil solicitar que algunos profesores, después de ver los videos, respondieran a dos preguntas:

a) Cuestión 1: Valore del 0 al 10 si recomendaría este video para resolver un problema inmediato, por ejemplo, una tarea de casa (aproximación a la eficacia puntual).

b) Cuestión 2: Valore del 0 al 10 si recomendaría este video para estudiar la asignatura (aproximación a la utilidad formativa).

Por la propia naturaleza de las preguntas, en ambos casos las respuestas tendrán un marcado carácter subjetivo, pero si las valoraciones de la primera cuestión son $x_{1}, x_{2}, \ldots, x_{N} \mathrm{y}$ de la segunda cuestión $y_{1}, y_{2}, \ldots, y_{N}$, conocer sus medias aritméticas $\bar{x}, \bar{y}$, respectivamente, y la diferencia entre ellas nos proporcionan una idea aproximada de los modos de enseñanza que estamos analizando:

$$
\begin{array}{ll}
\bar{x}=\frac{1}{N} \sum_{i=1}^{N} x_{i} & \Rightarrow \text { Estimación de la eficacia puntual, } \\
\bar{y}=\frac{1}{N} \sum_{i=1}^{N} y_{i} & \Rightarrow \text { Estimación de la utilidad formativa, } \\
|\bar{x}-\bar{y}| & \Rightarrow \text { Estimación equilibrio formativo. }
\end{array}
$$

Estas estimaciones no tienen por qué tener validez estadística para inferir conclusiones, pero lo cierto es que permiten reforzar el análisis cualitativo con el que se analizaron los modos de enseñanza, enfatizando los aspectos relacionados con la Representación de la información, el Esquema de pensamiento promocionado y la Consigna dominante. Por otra parte, somos concientes de que mientras $\bar{x}, \bar{y}$, tienen un carácter informativo en sí mismas, $|\bar{x}-\bar{y}|$ no puede utilizarse de forma aislada, puesto que debe observarse junto con las otras dos. De otro modo, podría suceder que para un video concreto, la diferencia de medias fuese muy baja (incluso nula) pero que las medias $\bar{x}, \bar{y}$ por separado fuesen números muy bajos, con lo cuál el uso de ese video estaría desaconsejado.

De manera similar a lo que ocurría con la valoración de las facetas didácticas (GODINO, 2013), para las que hemos expuesto la necesidad de analizar de forma cualitativa las razones 
por las que se han dado determinadas valoraciones numéricas, para el análisis del modo de enseñanza, nuestra propuesta es la misma. Tras la aproximación cuantitativa que acabamos de describir, el análisis de los videos con respecto a la eficacia puntual, la utilidad formativa y el equilibrio entre ellas se llevó a cabo a través de dos rubros: el tratamiento de la información y el esquema de pensamiento promocionado.

\section{Resultados y Análisis}

Para poner en práctica nuestra propuesta metodológica, se ha solicitado a 10 profesores de Matemáticas que visualicen los videos V1, V2 y V3 (todos ellos relacionados con la solución de ecuaciones lineales) proporcionen información de dos tipos: una respuesta cualitativa a los indicadores empíricos mencionados en la Sección 4.1 y una respuesta numérica y cualitativa a las cuestiones 1 y 2 de la Sección 4.2. En el siguiente cuadro recogemos, de forma breve, el análisis de los videos de cada faceta didáctica.

\begin{tabular}{|c|c|}
\hline \multicolumn{2}{|r|}{ Indicador empírico: ¿Contenido matemático correcto? - Faceta epistémica } \\
\hline Julioprofe & $\begin{array}{l}\text { Se identifica la incógnita, las operaciones se asocian a los datos disponibles. } \\
\text { Incorpora iconos y gráficos para apoyar la memoria. }\end{array}$ \\
\hline Unicoos & $\begin{array}{l}\text { Se identifica la incógnita, se proponen las operaciones necesarias para resolver el } \\
\text { problema con los datos disponibles. Uso de esquemas para dar sentido a las relaciones. }\end{array}$ \\
\hline Lasmatemáticas & $\begin{array}{l}\text { Presentación de los símbolos participantes. Tratamiento escrupuloso del contenido } \\
\text { matemático, desarrollado paulatinamente. }\end{array}$ \\
\hline \multicolumn{2}{|r|}{ Indicador empírico: ¿Aproximación didáctica adecuada? - Faceta cognitiva } \\
\hline Julioprofe & Tratamiento ordenado y enfatizando procedimientos, uso de reglas nemotécnicas. \\
\hline Unicoos & $\begin{array}{l}\text { Se usan esquemas que vinculan el problema y la ecuación. Un caso particular sirve para } \\
\text { justificar el caso general, se advierte de posibles interpretaciones erróneas. }\end{array}$ \\
\hline Lasmatemáticas & $\begin{array}{l}\text { Introducción progresiva de símbolos y operaciones y propiedades requeridas, justifica } \\
\text { procedimientos de despeje. }\end{array}$ \\
\hline \multicolumn{2}{|r|}{ Indicador empírico: ¿Son amenos, atractivos? - Faceta interaccional } \\
\hline Julioprofe & $\begin{array}{l}\text { Los movimientos corporales enfatizan el orden de los procedimientos, lo que orienta } \\
\text { claramente los pasos a seguir. Se muestra como profesor eficiente y ordenado. }\end{array}$ \\
\hline Unicoos & $\begin{array}{l}\text { El proceso del lenguaje es claro y coloquial. Enfatiza con el movimiento corporal. Se } \\
\text { muestra como profesor preocupado por ser accesible. }\end{array}$ \\
\hline Lasmatemáticas & $\begin{array}{l}\text { Proceso dilatado de construcción de la ecuación lineal tratada con cuidado y rigor, se } \\
\text { muestra como profesor persistente y conocedor de la materia. }\end{array}$ \\
\hline \multicolumn{2}{|r|}{ Indicador empírico: ¿Saca provecho del tiempo? - Faceta mediacional } \\
\hline Julioprofe & $\begin{array}{l}\text { Sí, se centra en la solución del problema planteado, incorpora exclusivamente la } \\
\text { información requerida por el problema. Discurso planificado. }\end{array}$ \\
\hline Unicoos & $\begin{array}{l}\text { Sí, la información adicional muestra posibles interpretaciones erróneas de los } \\
\text { resultados, se comentan sobre la pizarra los errores eventuales. Discurso planificado en } \\
\text { principio, con ramificaciones }\end{array}$ \\
\hline Lasmatemáticas & $\begin{array}{l}\text { Sí, pero en cada video, el tratamiento completo es dilatado. Trata todas las } \\
\text { eventualidades. }\end{array}$ \\
\hline \multicolumn{2}{|r|}{ Indicador empírico: ¿El discurso es amigable y ameno? - Faceta afectiva } \\
\hline Julioprofe & El nivel de empatía es neutro, pero la eficiencia, la gesticulación y el énfasis lo subsanan. \\
\hline Unicoos & $\begin{array}{l}\text { El discurso y la posición empática permiten dejar de lado cierto desorden de la } \\
\text { presentación. }\end{array}$ \\
\hline Lasmatemáticas & $\begin{array}{l}\text { Discurso neutro y consistente, sólo audio y un marcador para enfatizar. Puede no ser } \\
\text { ameno para un gran número de estudiantes. }\end{array}$ \\
\hline
\end{tabular}




\begin{tabular}{|l|l|}
\hline \multicolumn{2}{|c|}{ Indicador empírico: ¿Tratamiento adecuado por grado? - Faceta ecológica } \\
\hline Julioprofe & Los ejercicios suelen presentarse como auto contenidos y adecuados para el nivel. \\
\hline Unicoos & Los ejercicios se tratan relacionados y adecuados para el nivel. \\
\hline Lasmatemáticas & $\begin{array}{l}\text { Los ejercicios se relacionan progresivamente dentro del nivel siguiendo variaciones } \\
\text { matemáticas. }\end{array}$ \\
\hline
\end{tabular}

Cuadro 2 - Valoración de videos mediante indicadores empíricos

Fuente: Elaboración propia

Desde el punto de vista de la valoración didáctica anterior, las producciones analizadas resultan adecuadas en todos los casos, no cometen errores matemáticos y enmiendan pequeños fallos en el momento, mantienen la atención del estudiante con la empatía del discurso, el énfasis en la gesticulación y las imágenes o la solución precisa a dificultades concretas. No hay omisiones en la información requerida en ninguna de las producciones y las explicaciones incluyen la información necesaria para resolver la tarea propuesta. Además, el discurso, en general, puede considerarse ameno y los contenidos se adaptan a los programas respectivos.

En la Tabla 1 se muestran las valoraciones proporcionadas por los profesores encuestados para las cuestiones 1 y 2.

Tabla 1 - Valoración de videos con indicadores numéricos

\begin{tabular}{|c|c|c|c|c|c|c|}
\hline & \multicolumn{2}{|c|}{ Lasmatematicas } & \multicolumn{2}{|c|}{ Julioprofe } & \multicolumn{2}{|c|}{ Unicoos } \\
\hline & $\mathrm{EP}$ & UF & EP & UF & $\mathrm{EP}$ & UF \\
\hline P. 1 & 10 & 10 & 8 & 6 & 5 & 9 \\
\hline P. 2 & 6 & 8 & 8 & 7 & 7 & 8 \\
\hline P. 3 & 7 & 9 & 9 & 6 & 8 & 6 \\
\hline P. 4 & 8 & 9 & 8 & 4 & 8 & 7 \\
\hline P. 5 & 8 & 8 & 9 & 6 & 7 & 5 \\
\hline P. 6 & 5 & 5 & 8 & 6 & 8 & 9 \\
\hline P. 7 & 7 & 9 & 8 & 5 & 9 & 4 \\
\hline P. 8 & 5 & 8 & 9 & 2 & 8 & 3 \\
\hline P. 9 & 8 & 8 & 9 & 3 & 8 & 6 \\
\hline P. 10 & 5 & 9 & 9 & 5 & 9 & 5 \\
\hline Media & 6.9 & 8.3 & 8.5 & 5 & 7.7 & 6.2 \\
\hline Equilibrio & \multicolumn{2}{|c|}{1.4} & \multicolumn{2}{|c|}{3.5} & \multicolumn{2}{|c|}{1.5} \\
\hline
\end{tabular}

Fuente: Elaboración propia

De acuerdo con los resultados expresados en la Tabla 1, los encuestados prefieren la producción de Julioprofe para la eficiencia puntual (con una media de 8.5, frente a 7.7 y 6.9 de las otros dos), y la de Lasmatematicas para la utilidad formativa (una media de 8.3, frente a un 5 y un 6.2). Sin embargo, si lo que se busca es que en el video haya un equilibrio entre ambos objetivos, Lasmatematicas y Unicoos presentan un resultado muy similar (un 1.4 y 1.5, respectivamente).

En el Cuadro 3 mostramos los resultados del análisis de los videos con respecto a los modos de enseñanza, desarrollado a través de dos rubros: 1. Tratamiento de la información, 2. Esquema de pensamiento. 


\section{Holístico-Analítico (HA): Eficacia puntual - Julioprofe}

Tratamiento de la información: Domina un tratamiento secuenciado de operaciones y relaciones, enfatizado con flechas y colores. Mención de leyes y definiciones requeridas en la secuencia. La solución es el resultado directo de la aplicación de los procedimientos.

Esquema de pensamiento: La adecuada secuencia es la clave de la solución, el camino a tomar requiere de algunos recursos nemotécnicos, las partes se enlazan para formar un todo auto-contenido como un problema tipo. Consigna dominante: resolver aquí y ahora.
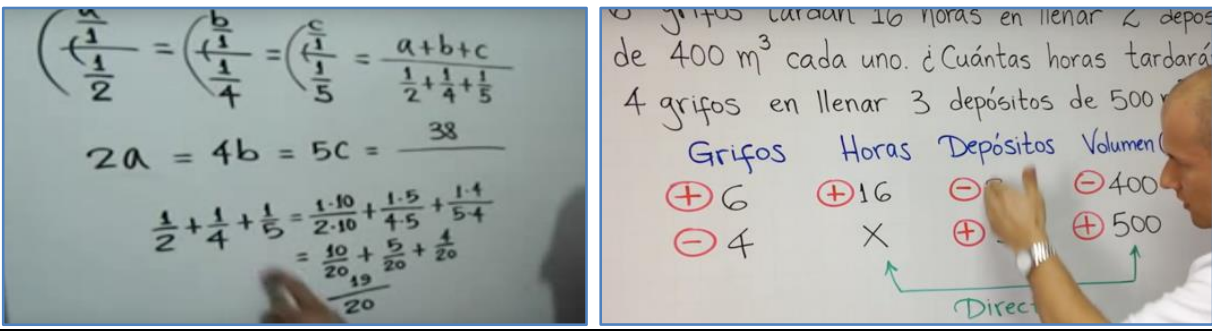

Verbal-Imaginativo (VI): Utilidad formativa - Unicoos

Tratamiento de la información: Desarrolla secuencias generales hasta encontrar situaciones que requieren de una explicación particular, entonces se hace uso de esquemas como sustento que da sentido a la situación que se aborda. En este video tenemos el caso de la fracción de una fracción, haciendo énfasis en la relación "parte de parte".

Esquema de pensamiento: El punto inicial es un problema central y se añaden otros periféricos, vinculados con frecuencia a través de imágenes mentales; también se usan gestos que sugieren imágenes metafóricas. Consigna dominante: resolver ahora y después.
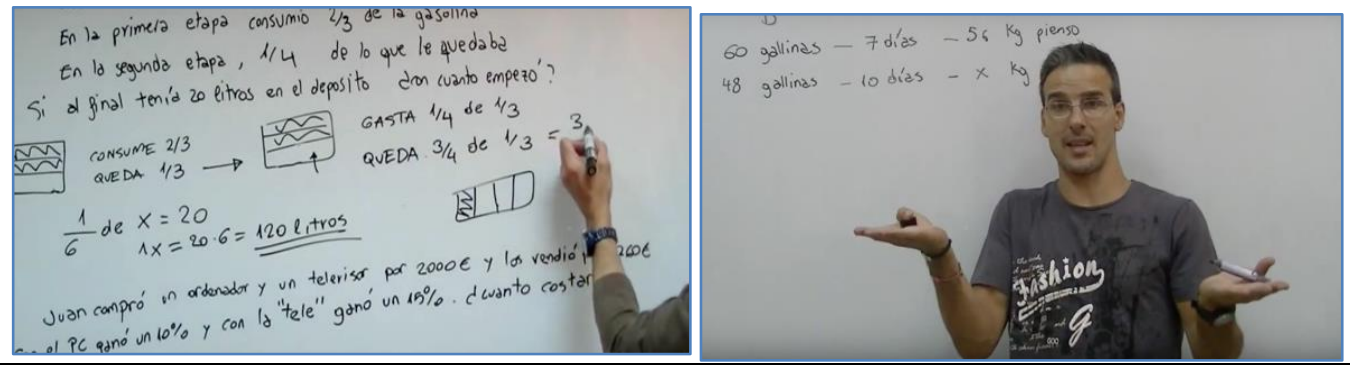

Equilibrio entre los modos de enseñanza - Lasmatemáticas.es

Tratamiento de la información: La información se trata como un proceso continuo, las flechas o rayas vinculan y enfatizan los elementos participantes, las imágenes propuestas son apoyadas con definiciones o resultados matemáticos que le dan sentido. Se atiende la solución rigurosamente.

Esquema de pensamiento: Los procesos de solución se apoyan en propiedades generales de la matemática que se mencionan explícitamente, como el tipo de soluciones o el cálculo del máximo común divisor. Consigna dominante: resolver ahora de forma general.

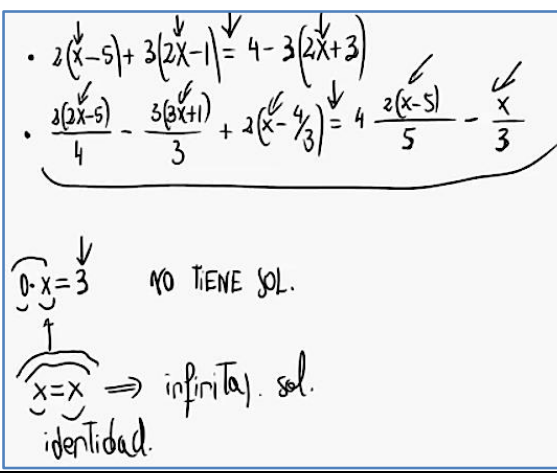

$$
\text { (4) } \begin{aligned}
\frac{2}{3} x-j & =\frac{1}{4} x-3 \\
\frac{2}{3} x-\frac{7}{4} x & =-3+1 \\
\frac{-13}{12} x & =-2 \\
x & =\frac{-2}{1}: \frac{-13}{12}=\frac{-24}{-13}=\frac{24}{13} \\
\frac{3}{3}-\frac{7}{4} & =\frac{8}{12}-\frac{21}{12}=\frac{-13}{12} \\
3 & =3 \quad \text { m.c. } m=3 \cdot 2^{2}=12 \\
4 & =2^{2}
\end{aligned}
$$

Cuadro 3 - Comparación de modos de enseñanza

Fuente: Elaboración propia

A partir del análisis recogido en el Cuadro 3, parece claro que los modos de enseñanza permiten diferenciar, tanto las habilidades de representación de la información como las 
consignas educativas particulares. En definitiva, cada modo de enseñanza puede resultar útil dependiendo de las necesidades del profesor, dado que no son incompatibles entre sí.

\section{Discusión}

Consideramos que los videos de matemáticas disponibles en internet pueden ser una fuente de recursos muy valiosa para apoyar la clase de matemáticas. Esta ayuda no se restringe al tratamiento de temas matemáticos fuera de clase, el tratamiento de aspectos que no son tratados en ella o la resolución de una tarea en casa, sino que estos materiales pueden reforzar de manera positiva el proceso de enseñanaza-aprendizaje.

Cuando nos cuestionamos sobre las aportaciones cognitivas de los videotutotiales de matemáticas a sus millones de usuarios, nos percatamos de claras diferencias que podrían contribuir a explicar su popularidad. Hemos analizado una pequeña muestra de la producción de tres de los autores de habla hispana más renombrados para mostrar el alcance de la idea de rúbrica de autor en las modalidades: eficacia puntual, utilidad formativa y equilibrio formativo. Esto nos ha permitido profundizar en las ventajas y posibles limitaciones de cada modo de enseñanza, dependiendo de las necesidades.

En la base del éxito de las producciones de estos autores, está el hecho de que cumplen con los requisitos didácticos para ser un referente confiable, como vimos con el análisis de indicadores empíricos, y esto los hace aconsejables para la educación matemática en general. Además, todos muestran una actitud razonablemente empática, ya sea enfatizando aspectos que son frecuentes obstáculos para los alumnos y alumnas o a través del tono comedido del ponente, quien cubre la necesidad de explicar los detalles que, por experiencia propia, sabe que resultan complejos.

Nuestro análisis de los modos de enseñanza ha mostrado grandes diferencias en los posibles efectos de cada aproximación. Las producciones de Julioprofe cubren con creces una necesidad urgente entre nuestros alumnos y alumnas de resolución de problemas, sin omisiones ni titubeos, se establece un orden de indicaciones, contribuye a representar la información como una estructura global formada por sus partes. Los videos del autor son apreciados por quienes requieren indicaciones directas, evitando el desvío de la atención. La consigna dominante es resolver aquí y ahora.

En el caso de Unicoos, la situación específica es abordada correctamente, al mismo tiempo que extiende su explicaciones a través del uso de imágenes visuales lo que, aún sin ser la intención, es posible vincularlo a resultados afines, ya que proporciona ligas funcionales. En 
este caso, en los problemas se mencionan posibles variaciones y hay un interés marcado por establecer afirmaciones con un alcance más general a partir de problemas particulares. Se trata de una propuesta adecuada para quienes requieren de una aproximación a la matemática de forma vinculada. La consigna dominante sería resuelve aquí y para después.

En Lasmatematicas parece buscarse intencionadamente el equilibrio formativo optando por una representación de la información armónica. Enfatiza en los procedimientos y definiciones matemáticos como partes de un todo encadenado, pero igualmente hace uso de una secuencia de imágenes mentales de carácter matemático, tratamiento que confiere al video un alto grado de rigor. Muchas de estas producciones requieren un seguimiento desde el inicio del tratamiento del tema, debido a que la información requerida se va acumulando. La consigna dominante en este caso es resuelve ahora con bases generales.

Más allá de las producciones analizadas, hemos propuesto un método para estimar la eficacia puntual, la utilidad formativa y el equilibrio formativo: Se valoran las dos primeras, mediante dos preguntas sencillas, y el equilibrio formativo puede aproximarse como la diferencia entre la eficacia puntual y la utilidad formativa. Es una manera sintética de comprobar si un video en cuestión sirve igualmente para aprender a resolver problemas o para estudiar la asignatura.

Por último, queremos comentar que, como decíamos al inicio de este trabajo, creemos que podemos contribuir a la tarea pendiente de analizar y valorar los videos que consumen nuestros alumnos y alumnas. Estudiar estos factores proporciona información sobre el tipo de enseñanza que es promovida, y este material puede ser útil para que el profesor tome sus decisiones sobre el video que eventualmente puede sugerir a sus estudiantes.

\section{Referencias}

ACUÑA-SOTO, C.; LIERN, V.; PÉREZ-GLADISH, B. A VIKOR-based approach for the ranking of mathematical instructional videos. Management Decision v. 57, n. 2, 2019, p. 501-522.

ACUÑA-SOTO, C.; LIERN, V.; PÉREZ-GLADISH, B. Multiple Criteria performance evaluation of YouTube mathematical educational videos by IS-TOPSIS. Operational Research, 2018a, p. 1-23.

ACUÑA-SOTO, C.; LIERN, V.; PÉREZ-GLADISH, B. Normalization in TOPSIS-based approaches with data of different nature: application to the ranking of mathematical videos. Annals of Operations Research, 2018b, p. 1-29.

BADILLO J.; FIGUEIRAS, L.; FONT, V.; MARTÍNEZ, M. Visualización gráfica y análisis comparativo de la práctica matemática en el aula. Enseñanza de las ciencias, Barcelona, v. 31, n. 3, 2013, p. 207-225. 
BREDA, A., FONT, V.; PINO-FAN, L. Criterios valorativos y normativos en la Didáctica de las Matemáticas: el caso del constructo idoneidad didáctica. Bolema, Rio Claro, v. 32, n. 60, 2018. p. 255-278

CASTRO, A.; PRAT, M.; GORGORIÓ, N. Conocimiento conceptual y procedimental en matemáticas: su evolución tras décadas de investigación. Revista de educación, España, 374, 2016 , p. 43-68, DOI: 10.4438/1988-592X-RE-2016-374-325.

CHEUNG, A.; SLAVING, R. The effectiveness of educational technology applications for enhancing mathematics achievement in K-12 classrooms: A meta-analysis. Educational research review, v 9, 2013, p. 88-113.

DONEVSK-TODOROVA, A.; TRGALOVA, J. Learning mathematics with technology, A review of recent CERME research. In: CONGRESS OF THE EUROPEAN SOCIETY FOR RESEARCH IN MATHEMATICS EDUCATION, 10, Dublin. Proceedings of 10 CERME, Dublin: Institute of Education, Dublin City University and ERME, 2017, p. 2539-2546.

GODINO, J.; BATANERO, C.; FONT, V. The onto-semiotic approach to research in mathematics, ZDM. The international journal on mathematics education, Germany, v. 39, n.1, 2, 2007. p. 127135 .

GODINO, J. D. Indicadores de la idoneidad didáctica de procesos de enseñanza y aprendizaje de las matemáticas. Cuadernos de investigación y formación en educación matemática, Costa Rica, v. 11,2013 p.111-132.

GUTIÉRREZ, A. Visualization in 3-dimensional geometry: In search of a framework. In: PUIG, L.; GUTIÉRREZ, A. (ed.). Proceedings of the 20th International Conference of the PME: vol. 1. Valencia: University of Valencia, 1996. p. 3-19.

HERSHKOWITZ, R. et al. Abstracting processes, from individuals' constructing of knowledge to a group's “shared knowledge”. Mathematics education research journal, Netherlands, v. 19, 2, 2007, p. 41-68.

HOHENWATER, J.; KOCADER, S.; HOHENWATER, M. Math Teachers' Adventure of ICT Integration: from an Open Online Course towards an Online Teacher Community. In: CONGRESS OF THE EUROPEAN SOCIETY FOR RESEARCH IN MATHEMATICS EDUCATION, 10, Dublin. Proceedings of the 10 CERME, Dublin: Institute of Education, Dublin City University and ERME, 2017, p. 2390-239.

KRUTETSKII V. The psychology of mathematical abilities in school children. Chicago University of Chicago Press, 1976.

KERSTING, N. Using video clips of mathematics classroom instruction as item prompts to measure teachers' knowledge of teaching mathematics. Educational and psychological measurement, v. 68, n. 5, 2008, p. 845-861.

LABIPE, Valoración de videotutoriales de matemáticas disponibles em internet con el modelo ValFM (Valoración Flexible Multicriterio), 2018. Disponible en: https://www.uv.es/liern/LABIPE.pdf Acceso: 13 nov. 2018.

LECHUGA, M.; MARTÍNEZ, Ú. Evaluación de vídeos de conceptos matemáticos elaborados por los estudiantes. Cultura y Educación, España, v.31, n. 4, 2019, p. 855-864.

MESSICK, S. The matter of style: Manifestations of personality in cognition, learning, and teaching. Educational psychologist, v. 29, n. 3, 1994, p. 121-136. 
PASK, G. Learning strategies, teaching strategies, and conceptual or learning style. In: SCHMECK, R. R. (ed.). Learning strategies and learning styles Boston: Springer, 1988, p. 83-100.

PITTA-PANTAZI, D.; CHRISTOU, C. Cognitive styles, dynamic geometry and measurement performance. Educational studies in mathematics, Netherlands, v. 70, n.1, 2009 p. 5-26.

PITTA-PANTAZI, D.; SOPHOCLEOUS, P.; CHRISTOU, C. Spatial visualizers, object visualizers and verbalizers: Their mathematical creative abilities. ZDM Mathematics education, Germany, v. 45, n. 2, 2013, p. 199-213.

PRESMEG, N. C. Visualization and mathematical giftedness. Educational studies in mathematics, Netherlands, v. 17, n. 3, 1986, p. 297-311.

RIVADENEIRA, F. Los canales de youtube y su aporte en el proceso de enseñanza aprendizaje. In: CONGRESO IBEROAMERICANO DE EDUCACIÓN MATEMÁTICA, 7, 2013, Montevideo. Actas del CIBEM, Montevideo: Biblioteca Nacional de Uruguay, 2013, p. 6923-6930.

ROMERO, R.; VÁZQUEZ, A.; GRAVÁN, P. YouTube: evaluación de un catálogo social de vídeos didácticos de matemáticas de calidad. Prisma Social: revista de investigación social, v. 18, 2017, p. 515-539.

SANTOS-MELLADO, J. et al. Use of math video tutorials. What are the users looking for? In: ANNUAL INTERNATIONAL CONFERENCE ON EDUCATION AND NEW LEARNING TECHNOLOGIES. Proceedings of the 9 EDULEARN, Granada, 2017, p. 8536-8541.

SANTOS MELLADO, J.; ACUÑA SOTO, C. Adaptación de los criterios de Idoneidad Didáctica para la valoración de videos educativos de matemáticas disponibles en Internet, In: CONTRERAS, J. M. et al. (ed.). Actas del segundo congreso Internacional Virtual sobre el Enfoque Ontesemiótico, Barcelona, 2017, p. 1-10

SANTOS MELLADO, J. Valoración de videotutoriales de matemáticas disponibles en internet. Nuevos instrumentos para el análisis de los procesos educativos. 2018, 123 p. Tesis (Doctorado en Educación Matemática) - Centro de Investigaciones y de Estudios Avanzados del IPN, México D.F.

SHERIN, M.; LINSENMEIER, K.; van ES, E. A. Selecting video clips to promote mathematics teachers' discussion of student thinking. Journal of Teacher Education, v. 60, n. 3, 2009, p.213-230.

SULLIVAN P.; MOUSLEY J. Thinking Teaching: Seeing Mathematics Teachers as Active Decision Makers. In: LIN, F. L.; COONEY T. (ed). Making sense of mathematics teacher education. Springer, Dordrecht, 2001, p.147-163

TALL, D. Teachers as Mentors to encourage both power and simplicity in active mathematical learning. In: ANNUAL CONFERENCE FOR MIDDLE EAST TEACHERS OF SCIENCE, MATHEMATICS AND COMPUTING, 3, Abu Dhabi. Proceedings of the 3 congress, Abu Dhabi: METSMaC, 2007, p. 13-28.

VIGOTSKY, L. S. Mind in society, Cambridge: Harvard University Press, 1978, 434 p.

ZABALZA, I. et al. Improving the teaching-learning process using educational videos as reusable learning objects (RLO) in the field of thermal engineering. In: INTERNATIONAL CONFERENCE ON EDUCATION AND NEW LEARNING TECHNOLOGIES, v. 8, 2016, Barcelona. Proceedings of the 8 EDULEARN, Barcelona: IATED, 2016, p. 363-372. 